\title{
Effects of miR-590 on oxLDL-induced endothelial cell apoptosis: Roles of p53 and NF-кB
}

\author{
MEI-HUA BAO ${ }^{1}$, JIAN-MING LI ${ }^{1}$, QI-LIANG ZHOU ${ }^{1}$, GUANG-YI LI ${ }^{1}$, \\ $\mathrm{JIE} \mathrm{ZENG}^{1}$, JUAN ZHAO ${ }^{1}$ and YI-WEN ZHANG ${ }^{2}$ \\ ${ }^{1}$ Department of Human Anatomy, Histology and Embryology, Institute of Neuroscience, Changsha Medical University, \\ Changsha, Hunan 410219; ${ }^{2}$ Department of Pharmacy, Zhejiang Cancer Hospital, Hangzhou, Zhejiang 310022, P.R. China
}

Received January 12, 2015; Accepted October 21, 2015

DOI: $10.3892 / \mathrm{mmr} .2015 .4606$

\begin{abstract}
Oxidized low-density lipoprotein (oxLDL)-induced endothelial cell apoptosis is considered to be important in atherogenesis. MicroRNA (miR)-590 has been reported to inhibit oxLDL-induced endothelial cell apoptosis. However, the mechanism underlying the inhibition of oxLDL-induced endothelial cell apoptosis by miR-590 remains to be elucidated. In the present study, the expression levels of miR-590 were quantified using reverse transcription-quantitative polymerase chain reaction analysis. Cell apoptosis was investigated using Hoechst staining and flow cytometry, and cell viability was measured using an MTS method. The protein expression levels of p53, B cell lymphoma 2 (Bcl-2), Bcl-2-associated X protein (Bax), caspase-3, lectin-like low-density lipoprotein receptor 1 (LOX-1), p38 mitogen-activated protein kinase (MAPK) and nuclear factor $(\mathrm{NF})-\kappa \mathrm{B}$ were quantified using western blot analyses. The results of the present study showed that oxLDL treatment inhibited the expression levels of miR-590 in a time-dependent and concentration-dependent manner. The overexpression of miR-590 inhibited oxLDL-induced endothelial cell apoptosis, expression of p53 and Bax, reduction of Bcl-2 and activation of caspase-3. miR-590 also inhibited the oxLDL-induced upregulation of the expression of LOX-1, overproduction of reactive oxygen species (ROS), phosphoryation of p38MAPK and translocation of NF- $\kappa$ B. These findings demonstrated the anti-apoptotic effects of miR-590 in oxLDL-treated endothelial cells, with the mechanisms underlying the effects of miR-590 involved, in part, in the LOX-1-ROS-p38MAPK-NF- $\mathrm{B}$ signaling cascade and the p53-Bcl-2/Bax-caspase-3 signaling pathway. The present study may provide novel insights into the protective properties of miR-590 in preventing atherosclerosis.
\end{abstract}

Correspondence to: Dr Mei-Hua Bao, Department of Human Anatomy, Histology and Embryology, Institute of Neuroscience, Changsha Medical University, $9 \mathrm{~km}$ Leifeng Road, Changsha, Hunan 410219, P.R. China

E-mail:mhbao78@163.com

Key words: microRNA-590, endothelial cell, oxidized low-density lipoprotein, atherosclerosis, p53

\section{Introduction}

Endothelial cells are monolayer cells located on the inner side of vessels, and the integrity and function of endothelial cells are considered important in the cardiovascular system (1). Oxidized low-density lipoprotein (oxLDL)-mediated endothelial cell apoptosis and dysfunction are reported to be closely associated with atherosclerosis through inflammatory processes and coagulation activity, eventually resulting in lesion rupture and clinical complications $(2,3)$.

MicroRNAs (miRs) are small non-coding RNAs, which regulate the expression of several proteins at the post-transcriptional level (4). In atherogenesis, several microRNAs, including miR-33, miR-133a and the let-7 family have been demonstrated to be important in atherogenesis (5-7). miR-590 is a microRNA located on chromosome $7 q 11.23$ (8). A previous study reported the anti-apoptotic effects of miR-590 on oxLDL-treated endothelial cells (9). The pilot studies also revealed a marked decrease in the expression levels of miR-590 in endothelial cells following oxLDL treatment. However, the mechanism underlying the inhibition of oxLDL-induced endothelial cell apoptosis by miR-590 remains to be elucidated.

Lectin-like low density lipoprotein receptor-1 (LOX-1) was first identified to be a receptor for oxLDL in endothelial cells (10). The upregulation in the expression of LOX-1 by oxLDL leads to the overproduction of reactive oxygen species (ROS), the phosphorylation of $\mathrm{p} 38$ mitogen-activated protein kinase (MAPK), the activation of nuclear factor (NF)- $\kappa \mathrm{B}$ and, eventually, endothelial activation, dysfunction and apoptosis (11). The LOX-1/ROS/p38MAPK/NF- $\kappa$ B signaling pathway is also involved in the protective effects of traditional Chinese medicines $(12,13)$. As upregulation in the expression of LOX-1 is considered to be the initial and crucial step in oxLDL-induced endothelial cell injury (14), the present study hypothesized that miR-590 may affect this signaling pathway, regulate apoptosis-associated protein expression and, eventually, prevent oxLDL-induced endothelial cell apoptosis. P53 is a tumor suppressor which modulates the apoptotic process by regulating the expression of $\mathrm{B}$ cell lymphoma 2 (Bcl-2) and Bcl-2 associated protein X (Bax) directly $(15,16)$. Bcl-2 and Bax are located upstream of caspase-3, a key enzyme in apoptosis signaling. The p53-Bcl-2/Bax-caspase-3 pathway serves an important role in apoptosis (17). 
Therefore, the aim of the present study was to examine the anti-apoptotic effects of miR-590 on oxLDL-treated endothelial cells, and to investigate the roles of the LOX-1/ROS/p38MAPK/NF- $\mathrm{B}$ and p53-B cell lymphoma 2 (Bcl-2)/Bcl-2-associated protein X (Bax)-caspase-3 apoptotic signaling pathways in these effects.

\section{Materials and methods}

Materials. The human umbilical vascular endothelial cell (HUVEC) line was obtained from the American Type Culture Collection (Manassas, VA, USA); oxLDL was supplied by the the Institute of Biochemistry, Peking Union Medical College (Beijing, China); a SYBR ${ }^{\circledR}$ Premix DimerEraser ${ }^{\mathrm{TM}}$ (Perfect Real Time) assay kit and a PrimeScript RT reagent kit with gDNA Eraser (Perfect Real Time) kit were purchased from Takara (Biotechnology Co., Ltd., Dalian, China); miR-590 mimics (5'-GAGCUUAUUCAUAAAAGUGCAG-3') and primers were obtained from Guangzhou RiboBio Co., Ltd. (Guangzhou, China); a lactate dehydrogenase (LDH) assay kit, Annexin V-fluorescein isothiocyanate (FITC) kit and dichloro-dihydro-fluorescein diacetate (DCFH-DA) were obtained from Beyotime Institute of Biotechnology (Shanghai, China); Invitrogen Lipofectamine ${ }^{\circledR} 2000$ transfection reagent was purchased from Thermo Fisher Scientific, Inc. (Waltham, MA, USA); rabbit polyclonal anti-LOX-1 antibody was purchased from Abcam (Cambridge, UK; cat. no. ab60178); mouse monoclonal anti-p38MAPK antibody (cat. no. sc-81621), mouse monoclonal anti-phosphorylated (p)-p38MAPK antibody (cat. no. sc-7973), mouse polyclonal anti-NF- $\mathrm{B}$ (p65) antibody (cat. no. sc-372), mouse monoclonal anti-p53 antibody (cat. no. sc-126), rabbit polyclonal anti-Bax antibody (cat. no. sc-526), rabbit polyclonal anti-Bcl-2 antibody (cat. no. sc-492), rabbit polyclonal anti-caspase-3 antibody (cat. no. sc-7148) and the goat anti-mouse (cat. no. sc-2005) and anti-rabbit (cat. no. sc-2004) IgG-horseradish peroxidase secondary antibodies were supplied by Santa Cruz Biotechnology, Inc. (Dallas, TX, USA).

Cell culture and transfection. As previously described (13), the HUVECs $\left(2 \times 10^{5}\right)$ were cultured in 6-well plates in complete medium [90\% Dulbecco's modified Eagle's medium supplemented with $10 \%$ fetal bovine serum (GE Healthcare Life Sciences, Logan, UT, USA)], and maintained in a humidified atmosphere containing $5 \% \mathrm{CO}_{2}$ at $37^{\circ} \mathrm{C}$.

For transfection, the endothelial cells were grown to $80 \%$ confluence. The media were then replaced with the transfection complexes and incubated for $6 \mathrm{~h}$. Subsequently, the medium was replaced with fresh normal growth medium, and incubated for a further $24 \mathrm{~h}$ at $37^{\circ} \mathrm{C}$.

The transfection complexes were obtained as follows: Lipofectamine ${ }^{\circledR} 2000(10 \mu 1)$, miR-590 mimics (7.5 $\mu 1$; $20 \mu \mathrm{M})$ or negative control (NC; $7.5 \mu \mathrm{l})$ mimics were diluted in $1 \mathrm{ml}$ Opti-MEM I Medium (Invitrogen; Thermo Fisher Scientific, Inc.) and incubated for $5 \mathrm{~min}$ at room temperature. Diluted Lipofectamine ${ }^{\circledR} 2000(1 \mathrm{ml})$ was mixed with diluted microRNA mimics (or NC mimics; $1 \mathrm{ml}$ ), and incubated for a further $20 \mathrm{~min}$ at room temperature.

Reverse transcription-quantitative polymerase chain reaction (RT-qPCR) detection of the expression levels of miR-590.
The HUVECs were seeded in 6-well plates at a density of $2 \times 10^{5}$ cells/well. The cells were then transfected with $25-75 \mathrm{nM}$ miR-590 for $24 \mathrm{~h}$ at $37^{\circ} \mathrm{C}$, as described above. Separate groups of HUVECs were treated with oxLDL $(150 \mu \mathrm{g} / \mathrm{ml})$ for different durations $(0,6,12$, and $24 \mathrm{~h})$ or at different concentrations $(50$, 100 and $150 \mu \mathrm{g} / \mathrm{ml}$ ) for $24 \mathrm{~h}$ at $37^{\circ} \mathrm{C}$.

Following incubation at $37^{\circ} \mathrm{C}$, total RNA was extracted using invitrogen TRIzol reagent (Thermo Fisher Scientific, Inc.), the concentration was measured using a BioPhotometer Plus (Eppendorf, Hamburg, Germany) by optical density at $260 \mathrm{~nm}$, and the purity of the RNA was evaluated at 260/A280. Reverse transcription reactions were performed using a PrimeScript RT reagent kit with a gDNA Eraser (Perfect Real Time) kit and an miR-590 stem loop RT primer. The PCR amplification reactions were performed using $2 \mu \mathrm{l}$ sample, $10 \mu \mathrm{l}$ SYBR Premix DimerEraser $^{\mathrm{TM}}$ (Perfect Real Time) assay kit, $0.6 \mu \mathrm{l}$ forward primer $(10 \mu \mathrm{M}), 0.6 \mu \mathrm{l}$ reverse primer $(10 \mu \mathrm{M})$ and $6.8 \mu \mathrm{l}$ water in a Roche LC480 PCR system (Roche Diagnostics, Basel, Switzerland). RT-qPCR was performed using the following thermocycling conditions: Initial incubation at $95^{\circ} \mathrm{C}$ for $30 \mathrm{sec}$, followed by 40 cycles of $95^{\circ} \mathrm{C} 5 \mathrm{sec}$, and $60^{\circ} \mathrm{C} 30 \mathrm{sec}$. All samples were run in triplicate, and U6 was used as an internal reference. The results were quantified using the $2^{(-\Delta \Delta \mathrm{Cq})}$ method (18).

Cell viability and detection of $L D H$ release. The HUVECs were transfected with $75 \mathrm{nM}$ miR-590 for $24 \mathrm{~h}$ at $37^{\circ} \mathrm{C}$, as described above. Following transfection, the cells were treated with $150 \mu \mathrm{g} / \mathrm{ml}$ oxLDL for a further $24 \mathrm{~h}$ at $37^{\circ} \mathrm{C}$. Cell viability was measured using the MTS method using the CellTiter $96^{\circledR}$ AQueous One Solution Cell Proliferation Assay (Promega Corporation, Madison, WI, USA), and LDH content in the medium was detected using an LDH assay kit, as previously described (12).

Cell apoptosis detection using flow cytometry (FCM) and Hoechst staining. The HUVECs were cultured and transfected, as described above. Following transfection, the cells were treated with oxLDL $(150 \mu \mathrm{g} / \mathrm{ml})$ for a further $24 \mathrm{~h}$ at $37^{\circ} \mathrm{C}$. Endothelial cell apoptosis was evaluated using FCM using the FC 500 MCL/MPL flow cytometer (Beckman Coulter, Inc., Brea, CA, USA) and Hoechst (Beyotime Institute of Biotechnology) staining, as previously described (13). For FCM, an Annexin V-FITC kit containing propidium iodide was used; the cells were harvested by trypsinization (Beyotime Institute of Biotechnology) and incubated with Annexin V-FITC and propidium iodide for $15 \mathrm{~min}$ at room temperature in the dark. Cell apoptosis rates were then analyzed and quantified using FCM. For the Hoechst 33342 staining, the cells were washed twice with phosphate-buffered saline (PBS), and incubated in $50 \mu \mathrm{l}$ Hoechst 33342 solution $(5 \mu \mathrm{g} / \mathrm{ml}$ in PBS) for $20 \mathrm{~min}$ in dark at room temperature following 15 min fixing with $4 \%$ paraformaldehyde (Beyotime Institute of Biotechnology). Morphological changes in the nuclear chromatin of the apoptotic cells were examined and analyzed using fluorescence microscopy (CX31; Olympus Corporation, Tokyo, Japan).

FCM analysis of the production of ROS. The HUVECs were cultured and transfected, as described above. Following transfection, the cells were treated with oxLDL $(150 \mu \mathrm{g} / \mathrm{ml})$ for a further $4 \mathrm{~h}$ at room temperature. Intracellular ROS production 
was measured using DCFH-DA, which was oxidized and transformed into high fluorescent dichlorofluorescin by intracellular ROS. Following treatment, the cells were incubated for $30 \mathrm{~min}$ at room temperature with DCFH-DA $(10 \mu \mathrm{M})$ at $37^{\circ} \mathrm{C}$. FCM was used to detect the fluorescence intensity.

Extraction of total, nuclear and cytosol proteins. The HUVECs were cultured and transfected, as described above. Following transfection, the cells were treated with oxLDL $(150 \mu \mathrm{g} / \mathrm{ml})$ for a further $24 \mathrm{~h}$. Following treatment, the cells were collected and washed twice with PBS. Total protein was extracted by adding $50 \mu \mathrm{l}$ radioimmunoprecipitation assay lysis buffer (Beyotime Institute of Biotechnology), containing $1 \%$ phenylmethanesulfonyl fluoride (PMSF) and $10 \mathrm{mM} \mathrm{NaF}$, to the cells for $30 \mathrm{~min}$.

For the cytosol and nuclear proteins, hypotonic or hypertonic lysis buffer (Beyotime Institute of Biotechnology) was used, respectively (12). The cytosol proteins were extracted using $200 \mathrm{ml}$ hypotonic buffer, containing $10 \mathrm{mM}$ Hepes (pH 7.9), $1.5 \mathrm{mM} \mathrm{MgCl}{ }_{2}, 10 \mathrm{mM} \mathrm{KCl}, 5 \mathrm{mM}$ dithiothreitol (DTT), $5 \mathrm{mM}$ PMSF, $10 \mu \mathrm{g} / \mathrm{ml}$ leupeptin, $1 \mu \mathrm{g} / \mathrm{ml}$ aprotinin and $1 \% \mathrm{NP}-40$, and incubated for $15 \mathrm{~min}$ at $4^{\circ} \mathrm{C}$. The lysates were then centrifuged at $12,000 \mathrm{xg}$ for $5 \mathrm{~min}$ at $4^{\circ} \mathrm{C}$, and the supernatants containing cytosol proteins were separated from the nuclei-containing pellet. The pellet was re-suspended and incubated in $100 \mathrm{ml}$ hypertonic buffer, containing $10 \mu \mathrm{M}$ Hepes, $5 \mathrm{mM} \mathrm{MgCl}_{2}, 12.5 \%$ glycerol, $0.2 \mathrm{M} \mathrm{NaCl}, 0.5 \mathrm{mM}$ DTT, $1 \mathrm{mM}$ PMSF, $10 \mu \mathrm{g} / \mathrm{ml}$ leupeptin and $1 \mu \mathrm{g} / \mathrm{ml}$ aprotinin, for $30 \mathrm{~min}$ at $4^{\circ} \mathrm{C}$. The lysates were subsequently centrifuged at $12,000 \mathrm{x}$ g for $10 \mathrm{~min}$ at $4^{\circ} \mathrm{C}$, and the supernatant containing the desired nuclear proteins were collected. The protein concentrations were measured using a bicinchoninic acid method (Beyotime Institute of Biotechnology).

Western blot analysis of the expression levels of LOX-1, p53, Bcl-2, Bax and caspase-3, phosphorlyation of p38 MAPK, nuclear translocation of $N F-\kappa B$ and degradation of $I \kappa B$. For the detection of LOX-1, p53, Bcl-2, Bax, caspase-3, p38MAPK, p-p38MAPK and I $\mathrm{B}, 40 \mu \mathrm{g}$ total proteins were separated by $12 \%$ SDS-PAGE and transferred onto polyvinylidene fluoride (PVDF) membranes (EMD Millipore, Billerica, MA, USA). The membranes were then incubated in blocking solution (4\% non-fat milk) for $1 \mathrm{~h}$ at room temperature, and subsequently incubated in 1:200 of the LOX-1, p53, Bcl-2, pro-caspase-3, caspase-3, p38MAPK, p-p38MAPK or IкB primary antibodies overnight at $4^{\circ} \mathrm{C}$. The membranes were washed and incubated with a 1:10,000 dilution of secondary antibody for $1 \mathrm{~h}$ at room temperature and were detected using an enhanced chemiluminescence system (ChemiDoc XRS; Bio-Rad Laboratories, Inc., Hercules, CA, USA). Relative intensities were analyzed using Quantity One ${ }^{\circledR}$ software, version 4.6.2.

For quantification of the expression levels of $\mathrm{NF}-\kappa \mathrm{B}$ the cytosol and nuclear extracts were separated using SDS-PAGE and transferred onto PVDF membranes. The membranes were then incubated in blocking solution (4\% non-fat milk) for $1 \mathrm{~h}$, followed by incubation with 1:200 of NF- $\kappa \mathrm{B}(\mathrm{p} 65)$ primary antibodies overnight at $4{ }^{\circ} \mathrm{C}$, washing with Tris-buffered saline and incubated in a 1:10,000 dilution of secondary antibody for $1 \mathrm{~h}$. Protein expression was detected using an enhanced chemiluminescence system (P0018; Beyotime Institute of
Biotechnology), and the relative intensities were analyzed using Quantity One ${ }^{\circledR}$ software. The relative percentage of $\mathrm{NF}-\kappa \mathrm{B}$ (nuclear)/NF- $\mathrm{B}$ (plasma) was determined to evaluate the nuclear translocation of $\mathrm{NF}-\kappa \mathrm{B}$.

Statistical analysis. All data were obtained from three independent experiments and are expressed as the mean \pm standard deviation. The significance of differences were analyzed using one-way analysis of variance or Student's t-test (unpaired). Multiple comparisons between groups were performed using Tukey's method. SPSS software, version 19.0 (SPSS, Inc., Chicago, IL, USA) was used to perform the statistical analysis. $\mathrm{P}<0.05$ was considered to indicate a statistically significant difference.

\section{Results}

Expression levels of miR-590 are altered following treatment with oxLDL and miR-590 transfection. As shown in Fig. 1A and $\mathrm{B}$, treatment with oxLDL $(150 \mu \mathrm{g} / \mathrm{ml})$ for 12 and $24 \mathrm{~h}$, or treatment with $100-150 \mu \mathrm{g} / \mathrm{ml}$ oxLDL for $24 \mathrm{~h}$ significantly inhibited the expression of miR-590, whereas treatment for a relatively short duration $(6 \mathrm{~h})$ or low concentration $(50 \mu \mathrm{g} / \mathrm{ml})$ of oxLDL had no effect on the expression of miR-590. These results suggested that miR-590 may function during the process of oxLDL injury. Following transfection of the HUVECs with various concentrations of miR-590 mimics, the expression levels of miR-590 in the cells increased significantly, in a concentration-dependent manner (Fig. 1C).

miR-590 has an anti-apoptotic effect on oxLDL-treated HUVECs. To examine the effects of miR-590 on oxLDL-induced HUVEC apoptosis, morphological changes in nuclear chromatin in the apoptotic cells were evaluated using Hoechst staining, and the apoptotic rates were measured using FCM. As shown in Fig. 2A, the cells in the NC group exhibited uniform blue chromatin staining with an organized structure, whereas oxLDL treatment resulted in apoptotic morphological changes, featuring bright blue, fluorescent, condensed nuclei and chromatin fragmentation under the fluorescence microscope, with increased frequency, compared with the NC group. The overexpression of miR-590 inhibited oxLDL-induced cell nuclear chromatin morphological alterations. The results of the FCM revealed that oxLDL treatment significantly increased the apoptotic rates of the cells $(3.55 \%$ for the NC group and $17.45 \%$ for the oxLDL group), and these increases were significantly inhibited by overexpression of miR-590 (Fig. 2B and $\mathrm{C}$ ). In addition, oxLDL treatment decreased cell viability and significantly increased LDH release, and these effects were attenuated by overexpression of miR-590 (Fig. 2D and E).

miR-590 reduces oxLDL-induced overproduction of ROS. To investigate the mechanisms underlying the anti-apoptotic effects of miR-590 on oxLDL-induced endothelial cell injuries, the ROS levels in the HUVECs were measured. As shown in Fig. 3, following $4 \mathrm{~h}$ treatment with oxLDL, the ROS levels in the HUVECs increased 2 -fold, compared with those in the NC group. This overproduction of ROS was reversed by miR-590, suggesting that ROS may be involved in the protective effects of miR-590. 


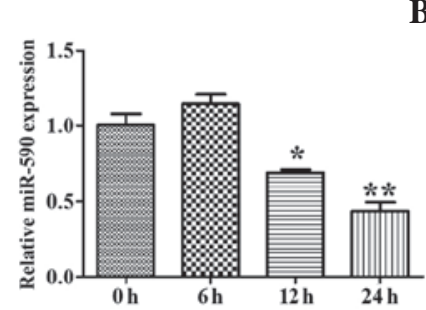

B

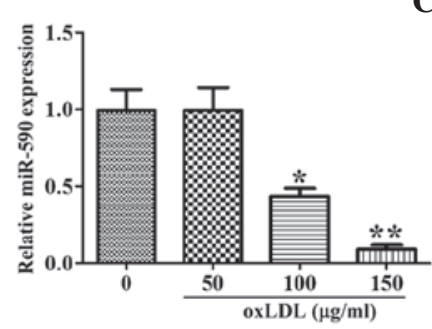

C

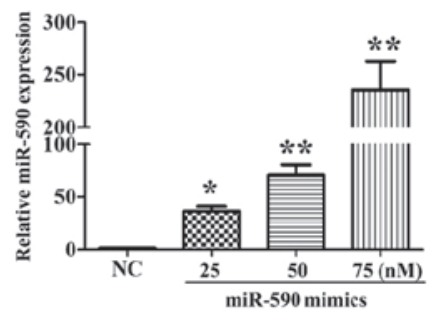

Figure 1. Effects of oxLDL on the expression of miR-590 and transfection efficiency in HUVECs. (A) Effects of $150 \mu \mathrm{g} / \mathrm{ml}$ oxLDL on expression levels of miR-590 at different time points. (B) Effects of different concentrations of oxLDL on expression levels of miR-590. (C) Relative expression levels of miR-590 in HUVECs following transfection with different concentrations of mimic for $48 \mathrm{~h}$. Data are presented as the mean \pm standard deviation of three independent experiments, and are relative to the NC, which was set as 1 . "P $<0.05$ and ${ }^{* *} \mathrm{P}<0.01$ vs. NC. oxLDL, oxidized low-density lipoprotein; HUVEC, human umbilical vascular endothelial cell; miR, microRNA; NC, negative control.

A

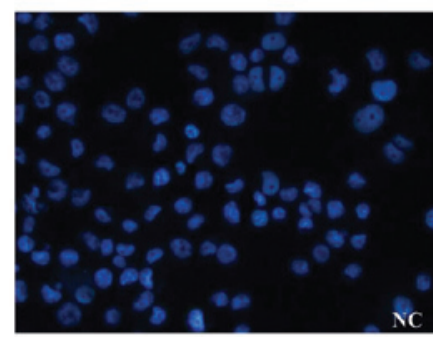

B

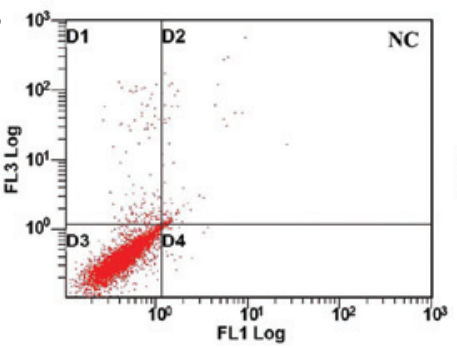

C

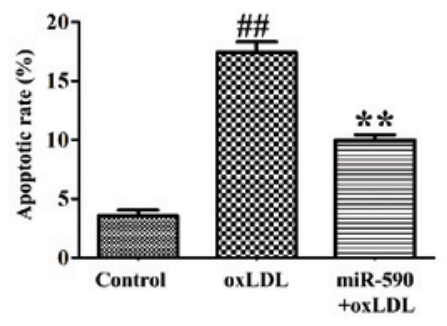

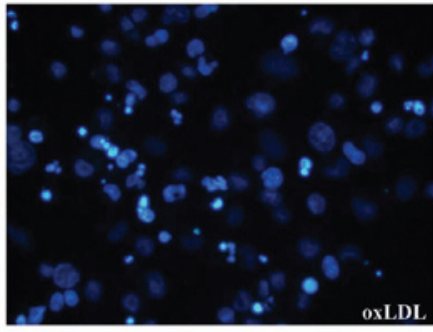

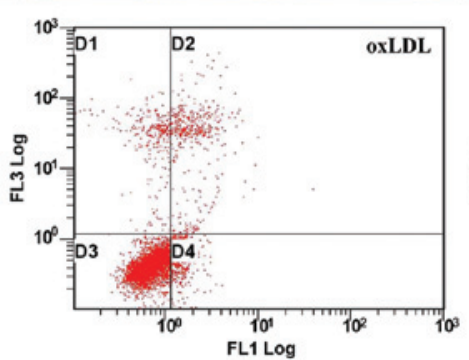

D

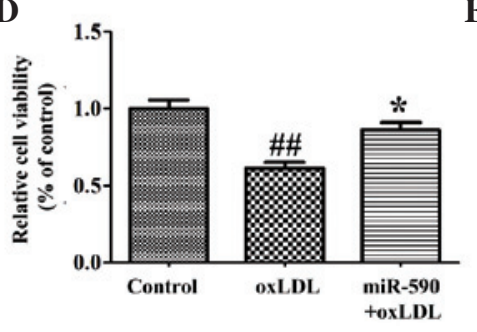

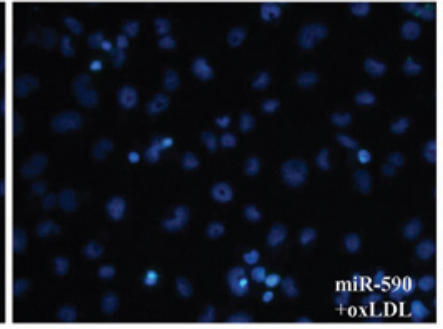

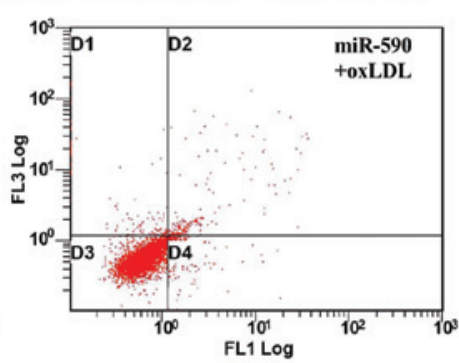

$\mathbf{E}$

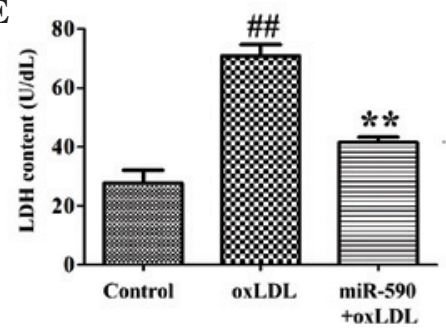

Figure 2. Effects of miR-590 on oxLDL-induced endothelial cell apoptosis, cell viability and LDH release. (A) Hoechst 33342 staining; magnification, x200. (B) FCM dot plots of apoptotic cells. The D1 quadrant indicates necrotic cells; the D2 quadrant indicates late stage apoptotic cells; the D3 quadrant indicates viable cells; and the D4 quadrant indicates early apoptotic cells. (C) Rates of apoptosis, quantified by FCM. (D) Relative cell viability. (E) LDH content in the cell medium. Data are presented as the mean \pm standard deviation of three independent experiments and are relative to the control. ${ }^{\# \#} \mathrm{P}<0.01$, vs. control; ${ }^{*} \mathrm{P}<0.05$ and ${ }^{* *} \mathrm{P}<0.01$, vs. oxLDL. oxLDL, oxidized low-density lipoprotein; miR, microRNA; FCM, flow cytometry.
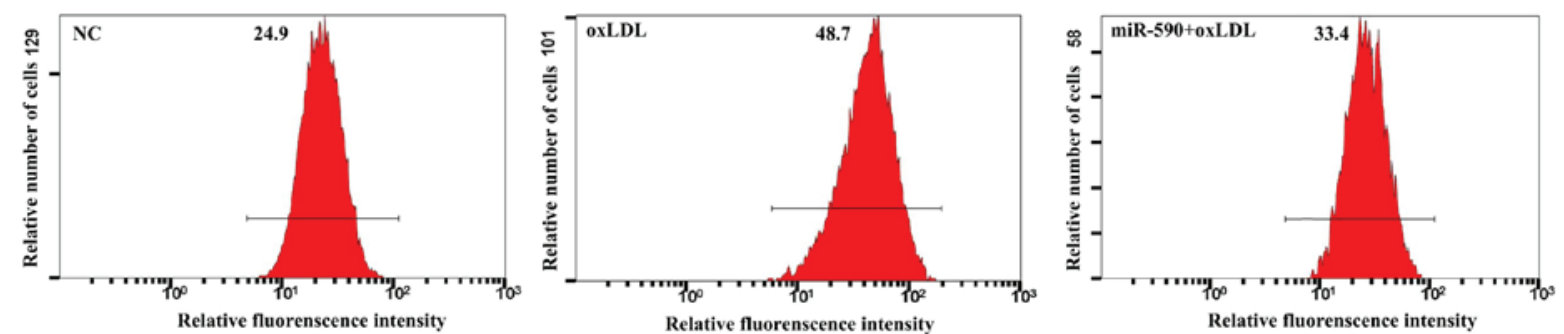

Figure 3. Effects of miR-590 on oxLDL-induced ROS production. HUVECs were transfected with miR-590 mimics ( $75 \mathrm{nM}$ ) for $24 \mathrm{~h}$, and the cells were then treated with oxLDL $(150 \mu \mathrm{g} / \mathrm{ml})$ for a further $4 \mathrm{~h}$. The intracellular ROS levels were measured using DCFH-DA staining and FCM detection. oxLDL, oxidized low-density lipoprotein; HUVEC, human umbilical vascular endothelial cell; miR, microRNA; NC, negative control; ROS, reactive oxygen species; FCM, flow cytometry; DCFH-DA, dichloro-dihydro-fluorescein diacetate. 
A

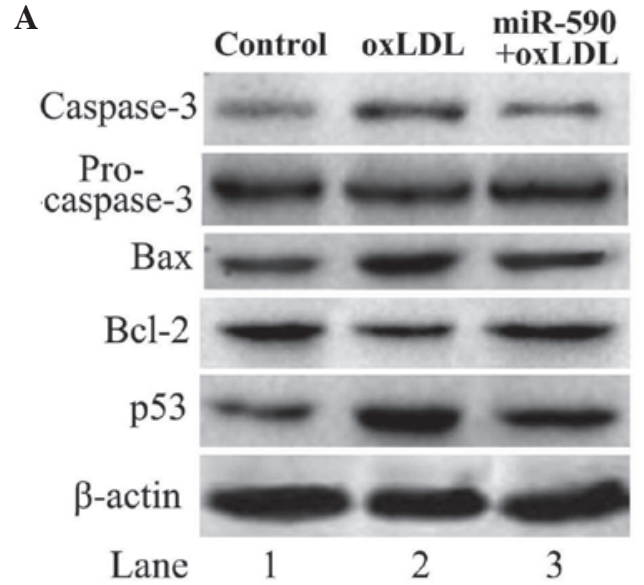

B

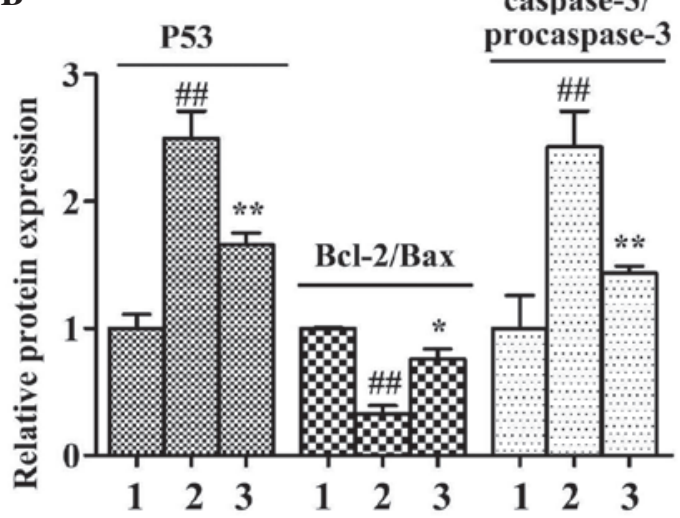

Figure 4. Effects of miR-590 on oxLDL-induced p53 induction, Bcl-2/Bax ratio reduction and caspase-3 activation. (A) Protein expression levels, determined using western blotting. (B) Relative band intensities, analyzed using Quantity One ${ }^{\circledR}$ software. Data are presented as the mean \pm standard deviation of three independent experiments and are are relative to the control, which was set as $1 .{ }^{\# \#} \mathrm{P}<0.01$, vs. control; ${ }^{*} \mathrm{P}<0.05$ and ${ }^{* *} \mathrm{P}<0.01$, vs. oxLDL. oxLDL, oxidized low-density lipoprotein; miR, microRNA; Bcl-2, B cell lymphoma 2; Bax, Bcl-2-associated X protein; 1, negative control; 2, oxLDL group; 3, miR-590 + oxLDL group.

Effects of miR-590 on the expression levels of oxLDL-induced p53, Bcl-2, Bax, and caspase-3. To determine which apoptosis-associated proteins were involved in the effects of miR-590, the expression levels of p53, caspase-3, Bcl-2 and Bax were quantified in the HUVECs. oxLDL treatment increased the expression levels of p53 and Bax and the activation of caspase-3, but inhibited the expression of Bcl-2. The ratio of $\mathrm{Bcl}-2 / \mathrm{Bax}$ was decreased to $32.7 \%$ of that in the control group (Fig. 4A and B). The overexpression of miR-590 significantly attenuated these effects.

Effects of miR-590 on oxLDL-induced upregulated expression of LOX-1, phosphorylation of p38MAPK, activation of $N F-\kappa B$ and degradation of $I \kappa B$. To determine the signals involved in the effects of miR-590, the phosphorylation of p38MAPK, activation of NF- $\kappa \mathrm{B}$ and expression levels of LOX-1 were measured in the HUVECs. The expression levels of LOX-1 were upregulated 2.05-fold, compared with the NC cells following treatment with oxLDL, as previously described $(12,14)$. By contrast, the overexpression of miR-590 reversed this upregulated expression of LOX-1 in the HUVECs (Fig. 5A).
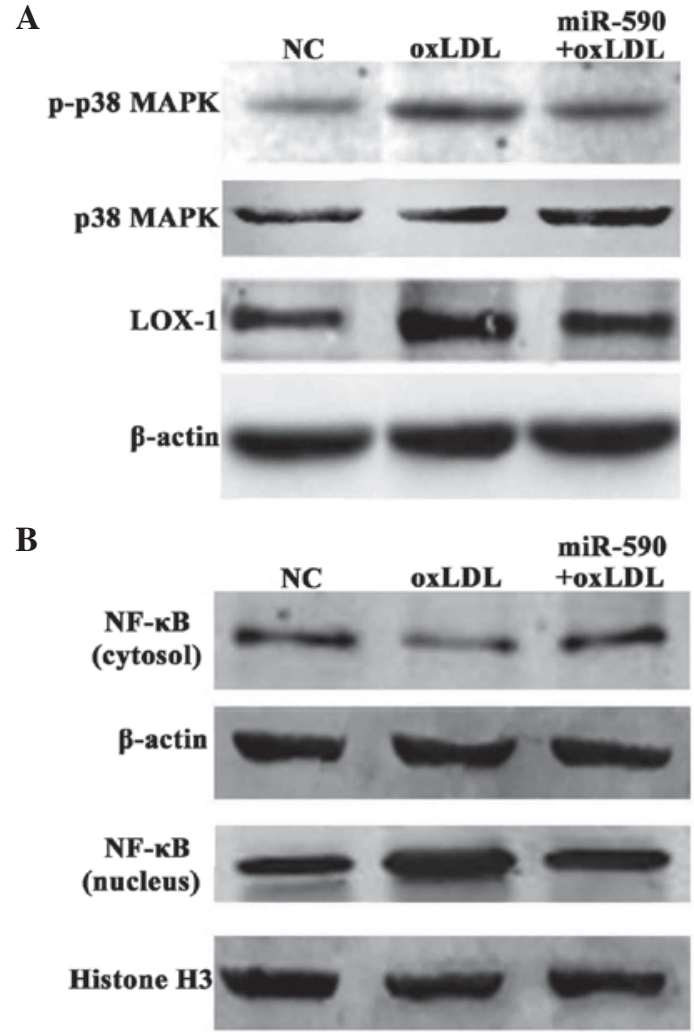

C
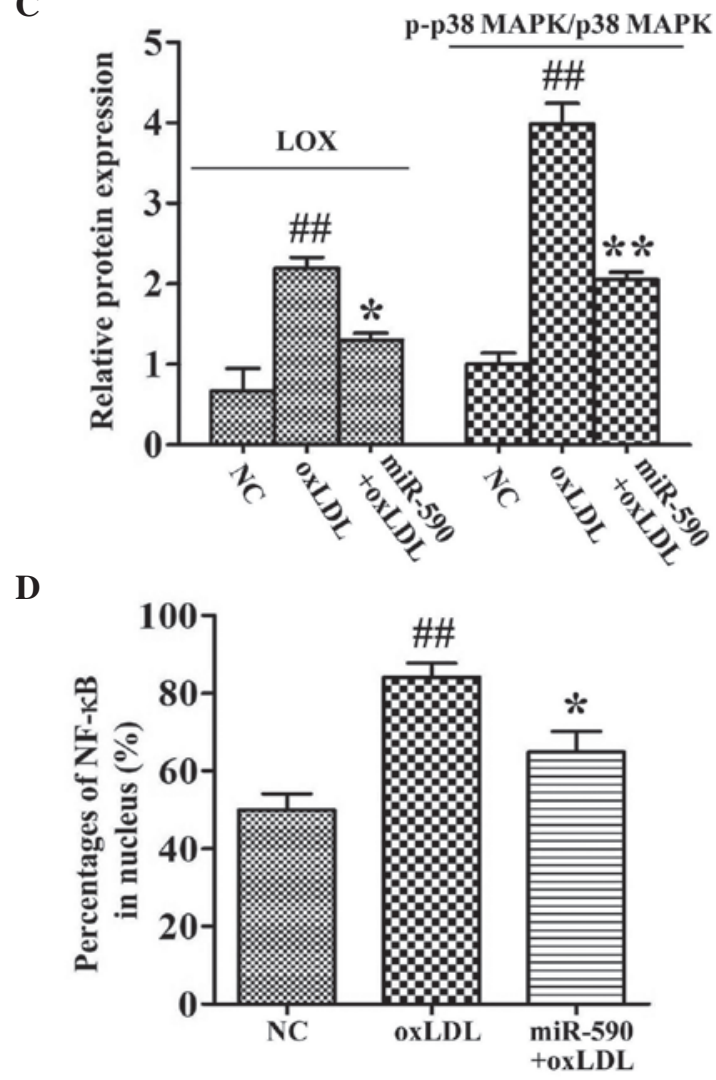

Figure 5. Effects of miR-590 on oxLDL-induced phosphorylation of p38MAPK, upregulation of LOX-1 and nuclear translocation of NF-kB. (A and B) Protein expression levels, determined using western blot analysis. $(C$ and $D)$ Relative band intensities, determined using Quantity One ${ }^{\circledR}$ software. Data are presented as the mean \pm standard deviation of three independent experiments and are relative to the control, which was set as 1. ${ }^{\# /} \mathrm{P}<0.01$, vs. control; ${ }^{\mathrm{P}} \mathrm{P}<0.05$ and ${ }^{* *} \mathrm{P}<0.01$, vs. oxLDL. oxLDL, oxidized low-density lipoprotein; miR, microRNA; p38MAPK, p38 mitogen-activated protein kinase; NF- $\kappa B$, nuclear factor- $\kappa \mathrm{B}$; LOX-1, lectin-like low-density lipoprotein receptor 1 ; $\mathrm{NC}$, negative control. 
OxLDL treatment also promoted the phosphorylation of p38MAPK (3.98-fold, compared with the oxLDL group). The expression levels of NF- $\mathrm{kB}$ in the nuclei also increased following oxLDL treatment $(49.8 \%$, vs. NC group; $84.1 \%$, vs. oxLDL group). The phosphorylation of p38MAPK and nuclear translocation of NF- $\mathrm{kB}$ were significantly inhibited by the overexpression of miR-590 (Fig. 5A-D).

\section{Discussion}

The present study demonstrated that oxLDL treatment inhibited the expression levels of miR-590 in a time- and concentration-dependent manner. The overexpression of miR-590 inhibited oxLDL-induced endothelial cell apoptosis. The mechanisms underlying the anti-apoptotic effects of miR-590 partly involved inhibiting the expression of LOX-1 and subsequent ROS generation, p38MAPK phosphorylation and NF- $\kappa \mathrm{B}$ activation. Apoptosis-associated proteins, including p53, Bcl-2, Bax and caspase-3 were also involved in the effects of miR-590.

Atherosclerosis is a severe vascular disorder, which results in several complications, including coronary artery disease, myocardial ischemia, cerebral ischemia and stroke (19). During the pathogenesis of atherosclerosis, oxLDL-induced endothelial cell apoptosis is key, and may lead to a reduction in vascular integrity, the deposition of lipids, invasion of vascular smooth muscle cells, migration of monocytes and formation of atherosclerotic plaques (20). In the present study, an increase in cell apoptosis following oxLDL treatment was observed, as previously reported (13). Notably, a time and concentration-dependent decrease in the expression levels of miR-590 was observed following oxLDL treatment. As the roles of several microRNAs in atherosclerosis have been elucidated in previous years $(21,22)$, the present study hypothesized that miR-590 may be involved in oxLDL-induced endothelial cell apoptosis. Therefore, the present study induced the overexpression of miR-590 prior to treatment of cells with oxLDL. As expected, the overexpression of miR-590 inhibited oxLDL-induced cell apoptosis. These results were consistent with previous reports (9), and suggested that miR-590 was involved in oxLDL-induced apoptosis. However, the mechanism underlying the protective effects of miR-590 remain to be elucidated, therefore, the present study investigated apoptosisassociated proteins and signaling cascades in oxLDL-treated endothelial cells.

p53 is a well-known tumor suppressor gene, which is located on chromosome 17p13.1 (23). Previous studies have investigated the roles of p53 in the apoptotic processes of several diseases, including atherosclerosis, myocardial ischemia, infarction and Alzheimer disease (24-27). In atherosclerosis, p53 has been demonstrated to be involved in endothelial cell injuries, vascular smooth muscle cell apoptosis and atherosclerotic plaque rupture (27). The present study investigated whether p53 was involved in the protective effects of miR-590, and observed a significant upregulation in the expression levels of p53 following oxLDL treatment in the endothelial cells, and this upregulation was inhibited by the overexpression of miR-590 . In addition to the changes in the expression of $\mathrm{p} 53$, the results also demonstrated a decrease in the $\mathrm{Bcl}-2 / \mathrm{Bax}$ ratio and an increase in caspase- 3 activation following oxLDL treatment, which was also attenuated by the overexpression of miR-590. p53 is a transcription factor, which regulates the expression levels of Bcl-2 and Bax directly at the transcriptional level $(15,16)$, and the upregulation of Bax subsequently promotes caspase- 3 activation. Therefore, the present study hypothesized that miR-590 may inhibit oxLDL-induced endothelial cell apoptosis via the p53-Bcl-2/Bax-caspase-3 pathway.

LOX-1 is an important receptor involved in oxLDL-induced cell apoptosis (14). Our previous study demonstrated the LOX-1-ROS-p38MAPK-NF- $\kappa$ B signals in oxLDL-treated endothelial cells (12). To examine whether these signals were involved in the effects of miR-590, the overexpression of miR-590 was induced in the present study, and the protein expression levels of LOX-1, production of ROS, phosphorylation of p38MAPK and nuclear translocation of NF- $\mathrm{KB}$ were quantified. The results demonstrated that miR-590 significantly inhibited the changes induced by oxLDL. As the interaction between oxLDL and LOX-1 is considered to be the initial step in oxLDL-induced endothelial cell injury, the inhibition of LOX-1 is regarded as beneficial for the prevention of atherogenesis (14). In the present study, a significant decrease in the expression of LOX-1 was observed following miR-590 overexpression, as previously reported (9). The present study also investigated whether miR-590 inhibited the expression LOX-1 directly using dual luciferase reporter assays. No effects of miR-590 on LOX-1 reporter gene expression levels were observed (data not shown). A previous study demonstrated that lipoprotein lipase (LPL) is a target for miR-590 in human THP-1 macrophages (28). Whether LPL is responsible for the downregulation in LOX-1 remains to be elucidated. Considering the complicated signaling networks and interactions in cells, the present study hypothesized that miR-590 downregulated the expression of LOX-1 in an indirect manner. However, which molecule is the target of miR-590, remains to be elucidated.

In the oxLDL-treated endothelial cells, the upregulation of LOX-1 promoted the overproduction of ROS, with subsequent p38MAPK phosphorylation and NF- $\kappa B$ activation. NF- $\mathrm{KB}$ is a multifunctional transcriptional factor, which regulates the expression of several apoptosis-associated genes, and several studies have demonstrated that the activation of NF- $\kappa \mathrm{B}$ is associated with the activation of caspase-3, induction of Bax and reduction of Bcl-2, either directly or indirectly (29-31). The present study demonstrated that miR-590 led to marked inhibition in the translocation of NF- $\mathrm{KB}$, which subsequently suppressed caspase-3 activation, Bax induction and the reduction of Bcl-2, and was responsible for the anti-apoptotic effects of miR-590.

p53 and NF- $\mathrm{kB}$ are transcription factors, which regulate the expression of certain apoptosis-associated genes, including Bcl-2 and Bax. However, the association between p53 and $\mathrm{NF}-\kappa \mathrm{B}$ remains controversial $(32,33)$. In cancer cells, NF- $\kappa \mathrm{B}$ and p53 antagonize the activity of one another (32), whereas, in oxLDL-treated endothelial cells, the expression levels of p53 and NF- $\mathrm{kB}$ are upregulated (33). The results of the present study demonstrated an increase in NF- $\kappa \mathrm{B}$ and p53 in the oxLDL-treated endothelial cells, and these effects were inhibited by the overexpression of miR-590. These results indicated that p53 and NF- $\mathrm{kB}$ may co-regulate the apoptosis 
of endothelial cells, and may be involved in the anti-apoptotic effects of miR-590.

In conclusion, the present study demonstrated that miR-590 exerted anti-apoptotic effects on oxLDL-treated endothelial cells. These anti-apoptotic effects of miR-590 were caused, in part, by the p53-Bcl-2/Bax-caspase-3/apoptotic pathway and the LOX-1-ROS-p38MAPK-NF- $\kappa$ B signaling cascade. The results of the present study may provide novel insights into the protective properties of miR-590 in the prevention of endothelial dysfunction associated with cardiovascular disease, including atherosclerosis.

\section{Acknowledgements}

The present study was supported by the Construct Program of the Key Discipline in Hunan Province, National Sciences Foundation of China (grant no. 81300231), and the Hunan Provincial Natural Sciences Foundation of China (grant no. 13JJ4112).

\section{References}

1. Pohl U, Holtz J, Busse R and Bassenge E: Crucial role of endothelium in the vasodilator response to increased flow in vivo. Hypertension 8: 37-44, 1986.

2. Jiang F, Gibson AP and Dusting GJ: Endothelial dysfunction induced by oxidized low-density lipoproteins in isolated mouse aorta: A comparison with apolipoprotein-E deficient mice. Eur J Pharmacol 424: 141-149, 2001.

3. Li R, Mittelstein D, Fang K, Beebe T, Quigley K, Berliner J and Hsiai TK: Angiopoeitin-2 modulates survivin expression in OxLDL-induced endothelial cell apoptosis. Biochem Biophys Res Commun 417: 619-622, 2012.

4. Lewis BP, Burge CB and Bartel DP: Conserved seed pairing, often flanked by adenosines, indicates that thousands of human genes are microRNA targets. Cell 120: 15-20, 2005.

5. Chen WJ, Zhang M, Zhao GJ, Fu Y, Zhang DW, Zhu HB and Tang CK: MicroRNA-33 in atherosclerosis etiology and pathophysiology. Atherosclerosis 227: 201-208, 2013.

6. Gao S, Wassler M, Zhang L, Li Y, Wang J, Zhang Y, Shelat H, Williams J and Geng YJ: MicroRNA-133a regulates insulin-like growth factor-1 receptor expression and vascular smooth muscle cell proliferation in murine atherosclerosis. Atherosclerosis 232: 171-179, 2014.

7. Bao MH, Zhang YW, Lou XY, Cheng $\mathrm{Y}$ and Zhou HH: Protective Effects of let-7a and let-7b on oxidized low-density lipoprotein induced endothelial cell injuries. PLoS One 9: e106540, 2014

8. Zhu XM, Han T, Sargent IL, Yin GW and Yao YQ: Differential expression profile of microRNAs in human placentas from preeclamptic pregnancies vs normal pregnancies. Am J Obstet Gynecol 200: 661.e1-7, 2009.

9. Qin B, Xiao B, Jiang T and Yang H: Effects of miR-590-5p on ox-LDL-induced endothelial cells apoptosis and LOX-1 expression. Journal of Central South University. Medical sciences 37: 675-681, 2012 (In Chinese).

10. Sawamura T, Kume N, Aoyama T, Moriwaki H, Hoshikawa H, Aiba Y, Tanaka T, Miwa S, Katsura Y, Kita T and Masaki T: An endothelial receptor for oxidized low-density lipoprotein. Nature 386: 73-77, 1997.

11. Li D and Mehta JL: Antisense to LOX-1 inhibits oxidized LDL-mediated upregulation of monocyte chemoattractant protein-1 and monocyte adhesion to human coronary artery endothelial cells. Circulation 101: 2889-2895, 2000.

12. Bao MH, Zhang YW and Zhou HH: Paeonol suppresses oxidized low-density lipoprotein induced endothelial cell apoptosis via activation of LOX-1/p38MAPK/NF- $\mathrm{BB}$ pathway. J Ethnopharmacol 146: 543-551, 2013.

13. Bao MH, Zhang YW, Lou XY, Xiao Y, Cheng Y and Zhou HH: Puerarin protects endothelial cells from oxidized low density lipoprotein induced injuries via the suppression of LOX-1 and induction of eNOS. Can J Physiol Pharmacol 92: 299-306, 2014.
14. Chen M, Masaki T and Sawamura T: LOX-1, the receptor for oxidized low-density lipoprotein identified from endothelial cells: Implications in endothelial dysfunction and atherosclerosis. Pharmacol Ther 95: 89-100, 2002.

15. Yamaguchi H, Chen J, Bhalla K and Wang HG: Regulation of bax activation and apoptotic response to microtubule-damaging agents by p53 transcription-dependent and -independent pathways. J Biol Chem 279: 39431-39437, 2004.

16. Wu Y, Mehew JW, Heckman CA, Arcinas M and Boxer LM: Negative regulation of bcl-2 expression by p53 in hematopoietic cells. Oncogene 20: 240-251, 2001

17. Borghetti G, Yamaguchi AA, Aikawa J, Yamazaki RK, de Brito GA and Fernandes LC: Fish oil administration mediates apoptosis of Walker 256 tumor cells by modulation of p53, Bcl-2, caspase-7 and caspase-3 protein expression. Lipids Health Dis 14: 94, 2015.

18. Livak KJ and Schmittgen TD: Analysis of relative gene expression data using real-time quantitative PCR and the 2(-Delta Delta C(T)) Method. Methods 25: 402-8, 2001.

19. Beckman JA, Creager MA and Libby P: Diabetes and atherosclerosis: Epidemiology, pathophysiology, and management. JAMA 287: 2570-2581, 2002.

20. Choy JC, Granville DJ, Hunt DW and Mcmanus BM: Endothelial cell apoptosis: Biochemical characteristics and potential implications for atherosclerosis. J Mol Cell Cardiol 33: 1673-1690, 2001.

21. Zhang T, Tian F, Wang J, Jing J, Zhou SS and Chen YD: Endothelial cell autophagy in atherosclerosis is regulated by miR-30-mediated translational control of ATG6. Cell Physiol Biochem 37: 1369-1378, 2015.

22. Bazan HA, Hatfield SA, O'Malley CB, Brooks AJ, Lightell D Jr and Woods TC: Acute loss of miR-221 and miR-222 in the atherosclerotic plaque shoulder accompanies plaque rupture. Stroke 46: 3285-3287, 2015.

23. Levne AJ: p53, the cellular gatekeeper for growth and division. Cell 88: 323-331, 1997.

24. Bennett MR, Littlewood TD, Schwartz SM and Weissberg PL: Increased sensitivity of human vascular smooth muscle cells from atherosclerotic plaques to p53-mediated apoptosis. Circ Res 81: 591-599, 1997.

25. Bialik S, Geenen DL, Sasson IE, Cheng R, Horner JW, Evans SM, Lord EM, Koch CJ and Kitsis RN: Myocyte apoptosis during acute myocardial infarction in the mouse localizes to hypoxic regions but occurs independently of p53. J Clin Invest 100: 1363-1372, 1997.

26. Alves da Costa C, Sunyach C, Pardossi-piquard R, Sévalle J, Vincent B, Boyer N, Kawarai T, Girardot N, St George-Hyslop P and Checler F: Presenilin-dependent gamma-secretase-mediated control of p53-associated cell death in alzheimer's disease. J Neurosci 26: 6377-6385, 2006.

27. Mercer J and Bennett M: The role of p53 in atherosclerosis. Cell Cycle 5: 1907-1909, 2006.

28. Khan MS, Halagowder D and Devaraj SN: Methylated chrysin induces co-ordinated attenuation of the canonical Wnt and NF-kB signaling pathway and upregulates apoptotic gene expression in the early hepatocarcinogenesis rat model. Chem Biol Interact 193: 12-21, 2011.

29. Kiang JG, Agravante NG, Smith JT and Bowman PD: 17-DMAG diminishes hemorrhage-induced small intestine injury by elevating Bcl-2 protein and inhibiting iNOS pathway, TNF- $\alpha$ increase and caspase-3 activation. Cell Biosci 1: 21, 2011.

30. Nakai M, Qin Z, Wang Y and Chase TN: NMDA and non-NMDA receptor-stimulated IkappaB-alpha degradation: Differential effects of the caspase-3 inhibitor DEVD.CHO, ethanol and free radical scavenger OPC-14117. Brain Res 859: 207-216, 2000.

31. Pal S, Bhattacharjee A, Ali A, Mandal NC, Mandal SC and Pal M: Chronic inflammation and cancer: Potential chemoprevention through nuclear factor kappa B and p53 mutual antagonism. J Inflamm (Lond) 11: 23, 2014.

32. Aoki M, Nata T, Morishita R, Matsushita $H$, Nakagami $H$, Yamamoto K, Yamazaki K, Nakabayashi M, Ogihara T and Kaneda Y: Endothelial apoptosis induced by oxidative stress through activation of NF-kappaB: Antiapoptotic effect of antioxidant agents on endothelial cells. Hypertension 38: 48-55, 2001.

33. Zhang L, Zhou G, Song W, Tan X, Guo Y, Zhou B, Jing H, Zhao S and Chen L: Pterostilbene protects vascular endothelial cells against oxidized low-density lipoprotein-induced apoptosis in vitro and in vivo. Apoptosis 17: 25-36, 2012. 